\begin{tabular}{|c|l|}
\hline Title & $\begin{array}{l}\text { Coherence between tissue oxygen indexes in vastus lateralis and gastrocnemius in repetition of impul se exercise with } \\
\text { high intensity }\end{array}$ \\
\hline Author(s) & Y ano, T.; Widjaja, W.; Shirakawa, K.; Lian, C-S; Xiao, Z.; Y unoki, T. \\
\hline Citation & $\begin{array}{l}\text { Acta physiologica hungarica, 102(2), 189-196 } \\
\text { https://doi.org/10.1556/036.102.2015.2.9 }\end{array}$ \\
\hline Issue Date & 2015-06 \\
\hline Doc URL & http://hdl.handle.net/2115/59553 \\
\hline Rights(URL) & http://creativecommons.org/icenses/by-nc/3.0/ \\
\hline Type & article \\
\hline File Information & Yano036.102.2015.2.9.pdf \\
\hline
\end{tabular}

Instructions for use 


\title{
Coherence between tissue oxygen indexes in vastus lateralis and gastrocnemius in repetition of impulse exercise with high intensity
}

\author{
Yano $\mathbf{T}^{1}$, Widjaja $\mathbf{W}^{2}$, Shirakawa $\mathrm{K}^{1}$, Lian C-S $\mathbf{S}^{1}$, Xiao $\mathbf{Z}^{1}$, Yunoki $\mathbf{T}^{1}$ \\ ${ }^{1}$ Department of Human Developmental Sciences, Faculty of Education, Hokkaido University, \\ Sapporo, Kita-ku, Japan \\ ${ }^{2}$ College of Sports Science and Technology, Mahidol University, Bangkok, Thailand
}

Received: July 4, 2014

Accepted after revision: January 7, 2015

\begin{abstract}
The purpose of this study was to determine whether tissue oxygen indices (TOIs) in two muscle groups oscillated and were synchronized in repetition of impulse exercise with high intensity. Five impulse exercises of 400 watts for $10 \mathrm{~s}$ were repeated with intervals of $6 \mathrm{~min}$. During this period, TOI was determined by near-infrared spectroscopy in the vastus lateralis and gastrocnemius muscles. TOIs in the two muscles oscillated at rest. The TOIs rapidly decreased during each impulse exercise and then recovered and overshot after each impulse. The TOIs oscillated during each interval period. During this test period, coherent and phase differences were determined. There was high coherence between TOIs in the two muscles with a peak value at $0.019 \mathrm{~Hz}$. There was a phase difference of $-45 \pm 32.4$ degrees between TOIs in the two muscles. This phase difference corresponded to about $6 \mathrm{~s}$ in time scale. It seemed from this time delay that impulse exercise was not a trigger factor for the starting point of TOIs in the two muscles. It has been concluded that TOIs oscillate and are synchronized between two muscles in repetition of impulse exercise with high intensity.
\end{abstract}

Keywords: coherence, phase difference, tissue oxygen index, impulse exercise, oscillation

It is well known that glycolysis is a dissipative structure that shows oscillation in yeast (3). Richard (11) reported a review of chemical oscillation in yeast. In an anaerobic condition, a chemical substance in glycolysis oscillates by glucose continual input with an interval of about $10 \mathrm{~min}$. By pulse input of glucose, NAD and NADH oscillate with a 37-sec interval, and their oscillations are gradually attenuated. Furthermore, when two yeast cell populations are mixed, the two oscillations are known to be synchronized. On the other hand, Satroutdinov et al. (12) described a different type of respiratory oscillation that is apparently not related to the cell. However, the cycle was around 40-50 min. Lloyd (6) has reported that there are three types of respiratory oscillation with periods of about one minute, about $40 \mathrm{~min}$ and one day. The time-keeping clock has been shown for oscillations of longer periods but not for oscillation of the shortest period in yeast.

In humans, it has been reported that phosphocreatine $(\mathrm{PCr})$ indicates damped oscillations during recovery from exercise (5) (that report is the first one to show a dissipative structure in which the order is self-organized in human skeletal muscle). This oscillation damps to resting level at 3-5 min during recovery. In visual inspection, the oscillation of PCr has

Corresponding author: Yano T

Department of Human Developmental Sciences, Faculty of Education

Hokkaido University, Sapporo, Kita-ku, Japan

Phone: 08-11-706-5090; E-mail: yano@edu.hokudai.ac.jp 
disappeared at 3-5 min during recovery. Since the oscillation of $\mathrm{PCr}$ recovery starts from the end of exercise, the damped oscillations in muscle groups that participated in whole body exercise may start at the same time from the end of exercise by the simultaneous termination of motions in the whole body.

The recovery of $\mathrm{PCr}$ is thought to be related to ATP supply in the mitochondrial membrane, in which the respiratory system operates (5). In order to supply ATP, the recovery of $\mathrm{PCr}$ requires consumption of oxygen in skeletal muscles, suggesting oscillation in the tissue oxygen index (TOI) in the muscle. If TOIs in muscle groups are triggered by the end of whole body exercise, TOIs will start oscillations at the same time. Furthermore, if the following oscillations of TOIs in two muscle groups are attracted to each other, TOIs may synchronize between the muscle groups. Naturally, the synchronization between the two muscle groups can occur with participation of the cardiorespiratory system as shown in a study on locomotor-respiratory coupling (10). However, since the oscillation of this coupling is much faster than the present metabolic one, locomotor-respiratory coupling is quantitatively different from metabolic synchronization, which was examined in the present study. There may be a different mechanism in this synchronization from that in locomotor-respiratory coupling.

The purpose of this study was therefore to determine whether TOIs in two muscle groups oscillated and were synchronized in repetition of impulse exercise with high intensity. In order to arrange similar starting points and rhythms of TOIs in two muscle groups, a strong and repeated impulse exercise was used as a trigger factor in the present study.

\section{Materials and Methods}

\section{Subjects}

Seven healthy males participated in this study. The mean and standard deviation of the ages, heights and body weights of the subjects were $24.7 \pm 3.0 \mathrm{yrs}, 173.0 \pm 7.7 \mathrm{~cm}$ and $69.3 \pm$ $12.4 \mathrm{~kg}$, respectively. Each subject signed a statement of informed consent following a full explanation regarding the nature of the experiment. The Ethics Committee of Hokkaido University Graduate School of Education approved the present study. This study was performed in accordance with the declaration of Helsinki.

\section{Experimental protocol}

Each subject performed a main test consisting of one impulse exercise and incremental exercise on a cycle ergometer (Ergometer $232 \mathrm{CXL}$, Combi, Tokyo, Japan). After resting for 5 min and $50 \mathrm{sec}$ on the cycle ergometer seat, 10-s impulse exercise with 400 watts work load at $80 \mathrm{rpm}$ was performed, and then the same impulse exercise was repeated five times with intervals of $6 \mathrm{~min}$. The work load of 400 watts is the maximal work rate of the cycle ergometer used in the present study. Incremental exercise was also performed at 50, 100, 150, and 200 watts for $5 \mathrm{~min}$ in each step, and the level was then increased by 20 watts for each 1 min until the subject could not maintain the revolution rate of pedaling $(60 \mathrm{rpm})$.

Before resting on the cycle ergometer seat prior to 400 watts impulse exercise, each subject sat on a chair to attach electrodes on the subject's chest for monitoring heart rate (HR) and to attach photo probes on the subject's left leg (vastus lateralis and gastrocnemius) for NIRS. Each subject was instructed to relax and to maintain cycle ergometer cranking in a horizontal position at rest and during recovery on the cycle ergometer. 


\section{Measurements and determinations}

Blood samples (each $100 \mu \mathrm{l}$ ) were collected from fingertips using a capillary tube. Each subject's hand was pre-warmed in $40-45^{\circ} \mathrm{C}$ water while sitting on the chair prior to each test in order to arterialize capillary blood. After this warming, the subject's hand was warmed by a heating glove at rest, during exercise and during recovery on the cycle ergometer. It has been shown that such blood samples might not accurately reflect arterial $\mathrm{O}_{2}$ pressure but can closely reflect arterial $\mathrm{CO}_{2}$ and $\mathrm{pH}(21)$. Samples were analyzed using a blood gas analyzer (i-STAT1, i-STAT, Abbott Point of Care Inc. IL, USA) to measure $\mathrm{CO}_{2}$ pressure $\left(\mathrm{PaCO}_{2}\right), \mathrm{pH}$ and lactate $(\mathrm{La})$.

Data for respiration gas exchange were obtained using a respiratory gas analyzer by the breath-by-breath mode (AEROMONITOR AE-310S, Minato Medical Science CO., LTD., Osaka, Japan). Ventilation (V்) was measured by a hot-wire flow meter, and the flow meter was calibrated with a syringe of known volume (2 liters). $\mathrm{O}_{2}$ and $\mathrm{CO}_{2}$ concentrations were measured by a paramagnetic oxygen analyzer and photometric gas analyzer, respectively. The gas analyzer was calibrated by known standard gas $\left(\mathrm{O}_{2}: 15.13 \%, \mathrm{CO}_{2}: 5.068 \%\right)$. Respiration gas exchange was measured continuously during rest, exercise, and recovery periods. Heart rate (HR) was recorded using a heart rate monitor installed in the respiratory gas analyzer. $\dot{\mathrm{V}}_{2}$ and HR were obtained breath-by-breath. In incremental exercise, breath-bybreath data were output as 20 -s data. In impulse exercise, breath-by-breath data were converted to1-sec data according to the method described below.

TOI in the left vastus lateralis and that in the left gastrocnemius were determined using a NIRS system (NIRO200x, Hamamatsu Photonics, K. K. Hamamatsu, Japan). Although NIRO200x can determine oxygenation and deoxygenation by the Modified Beer-Lambert method, TOI determined by the spacially resolved spectroscopy (SRS) method was used in the present study. The NIRS probe consisted of a light source and an optical detector, with a distance of $3.0 \mathrm{~cm}$ between the light source and detector. Triple-wavelength light $(735,810$ and $850 \mathrm{~nm}$ ) emitted from the light source penetrates tissue, where it is either absorbed or scattered, and some of the scattered light returns to the optical detector. The sampling frequency of TOI was $1 \mathrm{~Hz}$. TOI was calculated from deoxygenation $(\mathrm{HHb})$ and oxygenation $\left(\mathrm{O}_{2} \mathrm{Hb}\right)$ determined by the SRS method using the following equation:

$$
\mathrm{TOI}=\mathrm{O}_{2} \mathrm{Hb} /\left(\mathrm{HHb}+\mathrm{O}_{2} \mathrm{Hb}\right)
$$

\section{Calculation and statistical analysis}

In a previous study (20), in order to obtain 1-s data, breath-by-breath data obtained in repeated exercise with a time interval were converted to 1-s data in each exercise, and the data obtained in each exercise were summed. However, in this method, the oscillation of measured data is eliminated by the summation. In order to avoid this effect, breath-by-breath data for $\dot{\mathrm{Vo}}_{2}$ were interpolated into 1-s data using a three-dimensional spline in the present study, but there is also a problem in this method. Higher frequency of oscillation than the respiration rate has no meaning.

The 1-s data for TOI in the test period were analyzed by fast Fourier transform (FFT). In order to visualize the data of low frequency, a low pass filter was used for $1-\mathrm{sec} \dot{\mathrm{V}}_{2}$ and 1 -sec TOI. The pass frequency was set below $0.05 \mathrm{~Hz}$. Coherence and phase difference between the two TOIs were calculated with 15 windows and $50 \%$ overlap.

Results are presented as means \pm standard deviations. Significant levels of blood $\mathrm{pH}$ and lactate from resting values were tested by Dunnett's method. The significant level was set at $p<0.05$. 


\section{Results}

One subject was very weak and could only do the incremental exercise up to 150 watts. For that subject, the impulse exercise was therefore carried out at 200 watts. Maximal work rate performed by incremental exercise was $239 \pm 59.0$ watts. The work load (400 watts including 200 watts for the above subject) in impulse exercise per this maximal work rate performed in incremental exercise was $159 \pm 31.0 \%$. Peak $\dot{V}_{o_{2}}$ was $2.77 \pm 0.78 \mathrm{l} / \mathrm{min}$. Peak $\dot{V}_{o_{2}}$ was obtained by the maximal value of 20 -s data converted from breath-by-breath data for each subject in incremental exercise.

Figure 1 shows the $\dot{\mathrm{V}}_{2}$ kinetics in the five repeated impulse exercises. Immediately after starting the impulse exercise, $\dot{\mathrm{V}}_{2}$ rapidly increased and then decreased. $\dot{\mathrm{V}}_{2}$ then re-increased and subsequently decreased exponentially. The 1-sec data for $\dot{V}_{o_{2}}$ were transformed by a low pass filter. This led to missing of the rapid increase in $\dot{\mathrm{V}}_{2}$ in impulse exercise, but it appeared that there were short-term oscillations for $\dot{\mathrm{V}}_{2}$ during recovery.

Figure 2 shows TOIs in the vastus lateralis and gastrocnemius. There were oscillations in the two TOIs before the first impulse exercise. The TOIs rapidly decreased in impulse exercise and in recovery after impulse exercise. The recovery in TOIs showed overshoots and oscillations. By using low pass filtering with $0.05 \mathrm{~Hz}$, damped oscillations appeared during the intervals of repeated impulse exercises.

Figure 3 shows coherence and phase difference between the two TOIs. The peak of coherence appeared at $0.019 \mathrm{~Hz}$ (wavelength of $52 \mathrm{~s}$ ). At this frequency, phase difference was $-45 \pm 32.4$ degrees. This phase difference corresponds to $6.5 \mathrm{~s}(52 \cdot 45 / 360$ : wavelength phase difference/degree of one cycle). The lowest degree appeared at $0.039 \mathrm{~Hz}$.

Table I shows blood $\mathrm{pH}$ and blood lactate at rest and at 5 and $10 \mathrm{~min}$ during the last recovery after the $5^{\text {th }}$ impulse exercise. Blood $\mathrm{pH}$ was significantly decreased at $5 \mathrm{~min}$, but there was no significant difference between blood $\mathrm{pH}$ at rest and that at $10 \mathrm{~min}$ during the last recovery. Blood lactate values at $5 \mathrm{~min}$ and $10 \mathrm{~min}$ during the last recovery were significantly higher than the resting value.
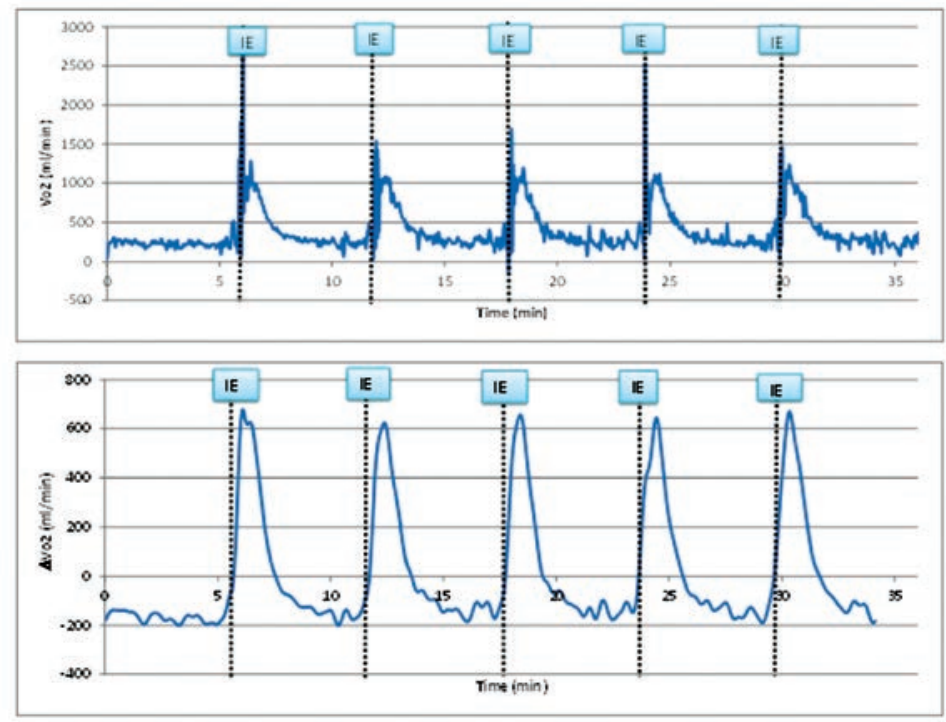

Fig. 1. An example of oxygen uptake $\left(\dot{\mathrm{V}}_{2}\right)$ in repeated impulse exercise (upper panel). An example of $\dot{\mathrm{V}}_{2}$ with a low pass filter for the same subject (lower panel). The dotted lines show impulse exercises (IE) 

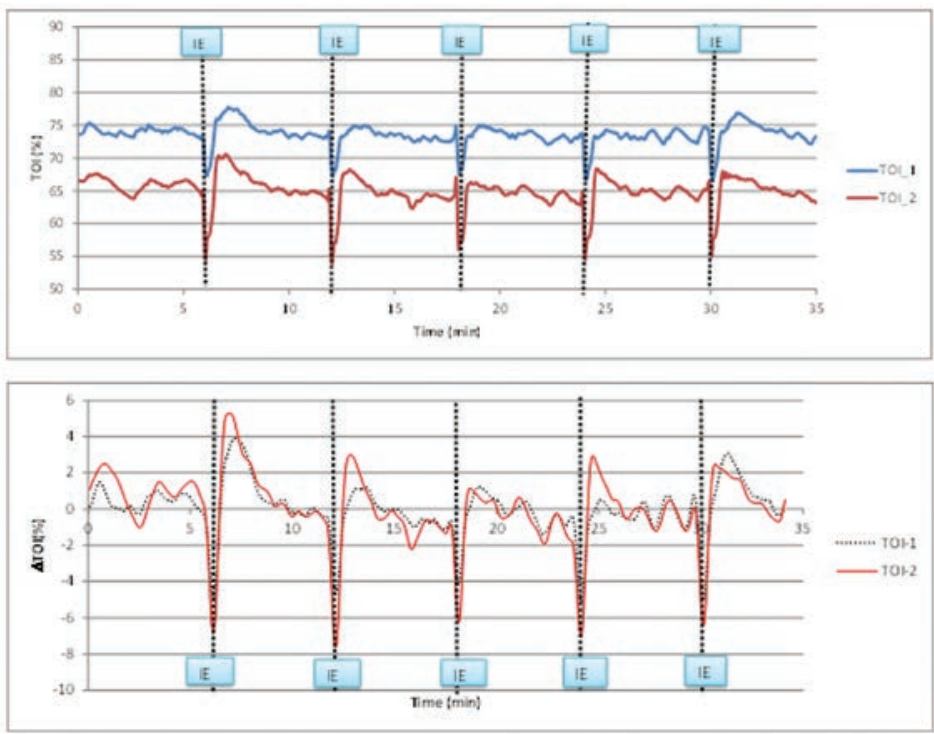

Fig. 2. Individual kinetics of tissue oxygen index in the vastus lateralis (TOI-1) and in the gastrocnemius (TOI-2) in repeated impulse exercise (upper panel). Examples of TOI-1 and TOI-2 with a low pass filter for the same subject (lower panel). The dotted lines show impulse exercises (IE)
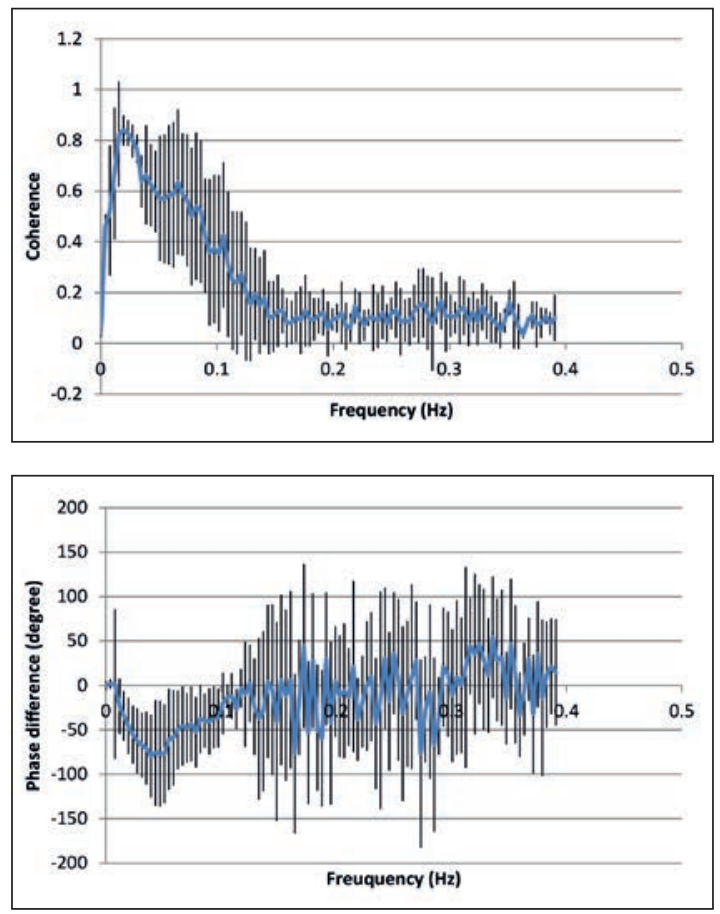

Fig. 3. Coherence (upper panel) and phase difference (lower panel) between tissue oxygen indices in the vastus lateralis and gastrocnemius 
Table I. Mean value and standard deviation (SD) of arterialized blood $\mathrm{pH}$ and blood lactate (La) at rest and during recovery after the $5^{\text {th }}$ impulse exercise

\begin{tabular}{|l|c|c|c|c|}
\hline & & Rest & Rec 5 min & Rec 10 min \\
\hline pH-5set & & & & \\
\hline Mean & & 7.411 & $7.365^{*}$ & 7.398 \\
\hline SD & & 0.037 & 0.020 & 0.021 \\
\hline La-5set & & & & \\
\hline Mean & $(\mathrm{mM})$ & 1.31 & $2.97^{*}$ & $2.34^{*}$ \\
\hline SD & & 0.30 & 0.97 & 0.77 \\
\hline
\end{tabular}

*: significant differences from resting values

\section{Discussion}

$\dot{\mathrm{V}}_{2}$ kinetics in the present study is similar to the results of a previous study on submaximal impulse exercise (4). The first increase in $\dot{\mathrm{V}}_{2}$ is thought to be related to an increase of cardiac dynamics (20). The second increase is known to be related to oxygen consumption in active muscle (20). It has been reported that $\dot{V}_{2}$ in impulse exercise is a nonlinear dynamic system (4). However, this did not mean that $\dot{\mathrm{V}}_{2}$ kinetics involved oscillation. Actual individual $\mathrm{Vo}_{2}$ kinetics at the lung level involves oscillation (15), though the oscillation is cancelled when $\dot{\mathrm{V}}_{2}$ is averaged. This oscillation in $\dot{\mathrm{V}}_{2}$ at the lung level suggests oscillation of oxygen consumption at the muscle level. This suggestion is supported by the finding of oscillation of deoxygenation in the muscle at rest and during light exercise $(17,18)$. Further support is provided by the finding that $\mathrm{PCr}$ can recover with damped oscillation from exercise (5), although PCr oscillation is found only in recovery. Thus, there would be an oscillation in mitochondria.

It has been shown that the oscillation of $\mathrm{PCr}$ during recovery ranged from $0.002 \mathrm{~Hz}$ to $0.025 \mathrm{~Hz}$ (5). The frequency in the present study was within this range. This range is dependent on cytosolic $\mathrm{pH}(\mathrm{pHc})$. The lower $\mathrm{pHc}$ is, the lower is the frequency. The present frequency corresponds to $7.0(=\mathrm{pHc})$ judging from the figure shown by Iotti et al. (5). During repetition of impulse exercise, blood $\mathrm{pH}$ decreased up to the end of the $5^{\text {th }}$ impulse exercise but increased during recovery, suggesting variation of $\mathrm{pHc}$ during the test period. Therefore, if the frequency of TOI oscillation is dependent on $\mathrm{pHc}$, some frequencies can be included. A peak frequency of coherence $(0.019 \mathrm{~Hz})$ is thought to be a representative one.

TOIs oscillated at rest in the present study, while it has been reported that $\mathrm{PCr}$ shows damped oscillation until 3-5 min during recovery, indicating no oscillation at rest (5). Although this TOI oscillation is supported by the finding of deoxygenation oscillation determined by NIRS not only at rest but also during exercise, there remains the problem of why there was a difference between the results determined by NIRS and those indicated by PCr.

Firstly, there is a methodological problem concerning the validity of NIRS. Accordingly, estimation of deoxygenation by the Beer-Lambert (BL) method in previous studies has been reported to be affected by skin blood flow under a certain condition $(1,2)$, whereas the validity of this method has been reported $(7,14)$. However, it is thought that estimation of TOI by spacially resolved spectroscopy (SRS), which was used in the present study is not greatly affected by oxygenation dynamics of skin blood flow (8). 
Secondly, there is a problem concerning the difference in mechanisms of oscillation. In yeast, NADH showed damped oscillation by a pulse input of glucose under the condition of cell starvation (6). However, in humans, homeostasis exists in the internal environment. Therefore, energy sources such as glucose, glycogen and free fatty acid are constantly given to the respiration system in skeletal muscle. This may lead to continuous oscillation of TOI. On the other hand, PCr shows damped oscillation despite continuous ATP supply. This may be caused by the upper limit of PCr production until the resting value.

Almost simultaneous discharge of the nervous system to the two muscle groups was hypothesized as a factor of synchronization of TOIs between the two muscles. In the present study, work load of impulse exercise was set so high that many muscle fibers of the muscle groups should be recruited. The rest of the recruitment leads to a continuous resting state of muscle fibers even in impulse exercise. In this case, impact cannot be given to oscillation of TOI in such muscle fibers. However, there was a phenomenon of sudden decrease in TOI in the two muscles in impulse exercise, suggesting that TOIs reflect the recruitment of muscle fibers in impulse exercise. Furthermore, there was a time delay between the two TOIs in the present study. This suggests that impulse exercise did not become a trigger factor of rearrangement of TOIs.

There was a time delay in synchronization between the two TOIs. The interaction may be derived from information through the nervous system or from humoral carriers from the lungs. However, interaction by the nervous system between the two muscles is not thought to make the synchronization since either the time delay was long.

One possible interaction is by a carrier of information transmitted by arterial blood. End tidal $\mathrm{CO}_{2}$ pressure $\left(\mathrm{PETCO}_{2}\right)$, which is controlled by the cardiorespiratory system, is one possible factor (16), since it was reported that there was an interaction between blood oxygen level-dependent (BOLD) in the brain and $\mathrm{PETCO}_{2}(9)$. However, it has been reported that there was no interaction between TOI in the muscle and $\mathrm{PETCO}_{2}$ since the number of oscillations of $\mathrm{PETCO}_{2}$ varied from person to person, while TOI oscillated with two waves (19).

We could not identify the carrier of information in this study, but there is definitely a carrier by arterial blood because high coherence was obtained and the peak frequency of coherence coincided with that within the range reported for $\mathrm{PCr}$ oscillation in the present study. This suggests that oscillation of a carrier, which is probably caused by the cardiorespiratory system, may be influenced by mitochondrial oscillation. Thus, there might be attraction of rhythm between the cardiorespiratory system and skeletal muscle system. Otherwise, we cannot explain the coincidence of the frequencies of TOIs and PCr.

Synchronization is a well-known fundamental phenomenon in nonlinear systems in natural science (13). Research of synchronization has proceeded in recent years. However, there has been a little investigation on metabolic oscillation in exercise physiology (19). It is concluded from the results of the present study that TOIs oscillate and are synchronized between the two muscles in the repetition of impulse exercise with high intensity. The oscillation of oxygenation determined by BL method was confirmed by TOI determined by SRS method. It seems that impulse exercise is not a trigger for the starting point of TOIs in the two muscles even if the exercise is repeated at high intensity. 


\section{REFERENCES}

1. Buono MJ, Miller PW, Hom C, Pozos RS, Kolkhorst FW: Skin blood flow affects in vivo near-infrared spectroscopy measurements in human skeletal muscle. Jpn. J. Physiol. 55, 241-244 (2005)

2. Davis SL, Fadel PJ, Cui J, Thomas GD, Crandall CG: Skin blood flow influences near-infrared spectroscopyderived measurements of tissue oxygenation during heat stress. J. Appl. Physiol. 100, 221-224 (2005)

3. De la Fuente IM, Cortes JM: Quantitative analysis of the effective functional structure in yeast glycolysis. PlosOne 7:e30162 (2012)

4. Hughson RL, Sherrill DL, Swanson GD: Kinetics of $\dot{V O}_{2}$ with impulse and step exercise in humans. J. Appl. Physiol. 64, 451-459 (1988)

5. Iotti S, Borsari M, Bendahan D: Oscillations in energy metabolism. Biochim. Biophys. Acta 1797, 1353-1361 (2010)

6. Loyd D: Respiratry oscillations in yeast. Adv. Exp. Med. Biol. 641, 118-140 (2008)

7. Mancini DM, Bolinger L, Li H, Kendrick K, Chance B, Wilso JR: Validation of near-infrared spectroscopy in humans. J. Appl. Physiol. 77, 2740-2747 (1994)

8. Messere A, Roatta S: Influence of cutaneous and muscular circulation on spatially resolved versus standard Beer-Lambert near-infrared spectroscopy. Physiol. Rep. 1:e00179 (2013)

9. Peng T, Niazy R, Payne SJ, Wise RG: The effects of respiratory $\mathrm{CO}_{2}$ fluctuations in the resting-state BOLD signal differ between eyes open and eyes closed. Magn. Reson. Imaging 31, 336-345 (2013)

10. Perségol L, Jordan M, Viala D: Evidence for the entrainment of breathing by locomotor pattern in human. J. Physiol. (Paris) 85, 38-43 (1991)

11. Richard P: The rhythm of yeast. Microbiology Reviews 27, 547-557 (2003)

12. Satroutdinov AD, Kuriyama H, Kobayashi H: Oscillatory metabolism of Saccharomyces cerevisiae in continuous culture. FEMS Microbiol. Lett. 77, 261-267 (1992)

13. Strogatz SH (2003): SYNC: The emerging science of spontaneous order. Brockman, Inc. New York

14. Tran TK, Sailasuta N, Kreutzer U, Hurd R, Chung Y, Mole P, Kuno S, Jue T: Comparative analysis of NMR and NIRS measurements of intracellular $\mathrm{PO}_{2}$ in human skeletal muscle. Am. J. Physiol. 276, R1682-R1690 (1999)

15. Yano T, Afroundeh R, Yamanaka R, Arimitsu T, Lian C-S, Shirakawa K, Yunoki T: Oscillation in $\mathrm{O}_{2}$ uptake in impulse exercise. Acta Pysiol. Hung. 101, 143-149 (2014)

16. Yano T, Afroundeh R, Yamanak R, Arimitsu T, Lian CS, Shirakawa K, Yunoki T: Response of end tidal $\mathrm{CO}_{2}$ pressure to impulse exercise. Acta Physiol. Hung. 101, 103-111 (2014)

17. Yano T, Lian CS, Arimitsu T, Yamanaka R, Afroundeh R, Shirakawa K, Yunoki T: Oscillation of oxygenation in skeletal muscle at rest and in light exercise. Acta Physiol. Hung. 100, 312-320 (2013)

18. Yano T, Lian C-S, Arimitsu T, Yamanaka R, Afroundeh R, Shirakawa K, Yunoki T: Comparison of oscillation of oxygenation in skeletal muscle between early and late phases in prolonged exercise. Physiol. Res. 62, 297-304 (2013)

19. Yano T, Widjaja W, Shirakawa K, Lian C-S, Yunoki T: Synchronization between tissue oxygen indices in the vastus lateralis and gastrocnemius after impulse exercise. Bulletin of Faculty of Education, Hokkaido University 121 , in press (2014)

20. Whipp BJ, Ward SA, Lamarra N, Davis JA, Wasserman K: Parameters of ventilatory and gas exchange dynamics during exercise. J. Appl. Physiol. Respir. Environ.Exerc. Physiol. 52, 1506-1513 (1982)

21. Zavorsky GS, Cao J, Mayo NE, Gabbay R, Murias JM: Arterial versus capillary blood gases: a meta-analysis. Respir. Physiol. Neurobiol. 155, 268-279 (2007) 